\title{
Nonhost Versus Host Resistance to the Grapevine Downy Mildew, Plasmopara viticola, Studied at the Tissue Level
}

\author{
A. M. Díez-Navajas, S. Wiedemann-Merdinoglu, C. Greif, and D. Merdinoglu
}

Unité Mixte de Recherche 1131, INRA-ULP Santé de la Vigne et Qualité du Vin, 28 rue de Herrlisheim, F-68021 Colmar cedex, France. Current address of first author: NEIKER-Tecnalia, Granja Modelo Arkaute, E-01080 Vitoria-Gazteiz, Spain. Accepted for publication 5 March 2008.

\section{ABSTRACT}

Díez-Navajas, A. M., Wiedemann-Merdinoglu, S., Greif, C., and Merdinoglu, D. 2008. Nonhost versus host resistance to the grapevine downy mildew, Plasmopara viticola, studied at the tissue level. Phytopathology 98:776-780.

Following inoculation of host and nonhost plants with Plasmopara viticola, the grapevine downy mildew, a histological survey was undertaken to identify the stage where its development is contained in nonhosts and in resistant host plants. Three herbaceous nonhost species, Beta vulgaris, Lactuca sativa, and Capsicum annuum, and three grapevine species displaying different level of resistance (Vitis vinifera [susceptible], Vitis riparia [partially resistant] and Muscadinia rotundifolia [totally resistant]) where inoculated by $P$. viticola using a controlled leaf disk inoculation bioassay. During the early steps of infection, defined as

Plants are permanently exposed to various potential pathogens but only a few such organisms establish close interactions with plants. To escape to potential pathogens, plants have evolved various constitutive and induced defense mechanisms leading to basic innate immunity. Nonhost resistance includes recognition of common microbial molecules as pathogen-associated molecular pattern (MAMPS or PAMPS) by pattern recognition receptors (PRRs) located in the plasma membrane (14). Fungal enzymes and cell wall fragments as well as host cell fragments are considered as nonspecific elicitors of plant defense against fungi. This surveillance system allows plant to sense non-self-molecules or host cellular intactness and to respond rapidly by various induced defense mechanisms. To establish infection, pathogens must evade, suppress, or manipulate host defenses by microbial effectors that act inside the plant cell (2). Effectors are defined as molecules that manipulate host cell structure and functions, thereby facilitating infection (virulence factors or toxins) and/or triggering defense responses (avirulence factors or toxins) (16).

Downy mildew oomycetes, powdery mildews, and rust fungi are phylogenetically unrelated groups of plant pathogens that use specialized infection strategies to take up nutrients from their host. They are obligate biotrophic organisms that depend exclusively on living plant cells for their growth and propagation (10). For exchange of nutrients and signal molecules with the host plant, obligate biotrophs produce specialized intracellular hyphal branches named haustoria (19). In most downy mildew oomycetes, ellipsoid to pyriform haustoria are produced at variable intervals along the intercellular hyphae. Haustorium development involves localized degradation of the plant cell wall and in-

Corresponding author: D. Merdinoglu; E-mail address: merdino@ colmar.inra.fr

doi:10.1094/PHYTO-98-7-0776

(C) 2008 The American Phytopathological Society encystment of zoospores on stomata, penetration of the germ tube, and production of the vesicle with the primary hypha, there was no evidence of a clear-cut preference to grapevine tissues that could attest to host specificity. The main difference between host grapevine species and nonhosts was observed during the haustorium formation stage. In nonhost tissues, the infection was stopped by cell wall-associated defense responses before any mature haustorium could appear. Defense responses in resistant grapevines were triggered when haustoria were fully visible and corresponded to hypersensitive responses. These observations illustrate that, for P. viticola, haustorium formation is not only a key stage for the establishment of biotrophy but also for the host specificity and the recognition by grapevine resistance factors.

Additional keywords: biotrophism, cytology, oomycete. vagination of the plasma membrane establishing a close relationship between host cells and the parasite $(8,29)$.

The cellular interactions which occur between biotrophic pathogens and host or nonhost plants have received considerable attention, especially for powdery mildews and rust fungi $(5,9,13$, $21,24-26,31)$ and more recently for oomycetes $(2,8,16)$. During compatible interactions, powdery mildew and rust fungi cause the suppression of host defenses at the haustorium-development stage, possibly as a consequence of biotroph-mediated reprogramming of host cells (24). Apart from the generalized induction of a hypersensitive response (HR), not much is known about nonhost versus host resistance to oomycetes. Cytological analyses revealed that penetration of epidermal cells occurred in all examined Phytophthora infestans-plant species interactions. In both resistant hosts and nonhost plants, an HR was triggered as soon as the primary hypha penetrated epidermal cells $(15,35)$. Most downy mildew oomycetes are not able to enter the intercellular spaces of the leaf mesophyll except via stomata where they form an infection vesicle with primary hypha and the first haustorium. This nonintrusive mode of entry is expected to have consequences on the timing of recognition of the pathogen by the plant cells and on the nature of induced defense responses. Thus, only the subsequent penetration of intact host cells by the haustorium leads to an intimate contact of the pathogen and its host. The outcome of plant-pathogen interaction seems to be determined at this intimate interface (14).

In this paper, a histological study of the early infection events of some host Vitis species by P. viticola (Berk. \& M. A. Curt.) Berl. \& de Toni, the grapevine downy mildew oomycete has been extended to include nonhost herbaceous plants species. To discover if that earlier stage of the infection process is revelatory of host specificity, the efficiency of attraction of $P$. viticola zoospores and germ tubes by host and nonhost stomata was compared. Further observations of internal infection structures were 
undertaken to determine the precise infection stage where $P$. viticola is recognized as a host or nonhost pathogen. For this purpose, $P$. viticola infection was investigated in six plant species: three nonhost herbaceous species (Beta vulgaris, Lactuca sativa, and Capsicum annuum) and three grapevine species displaying different resistance levels to the pathogen (the susceptible Vitis vinifera, the partially resistant Vitis riparia (30) and the totally resistant Muscadinia rotundifolia (7). This latter southeastern United States native grapevine species is closely related to Vitis species and presents an overall resistance to the main grapevine pathogens and pests (7). This study should help to identify the exact step of the infection process when the obligate biotroph $P$. viticola starts to undergo differential responses at the tissue level with respect to host and nonhost plant species and to learn how these differential responses are linked to the biological bases of the parasite biotrophism.

\section{MATERIALS AND METHODS}

Plant material and pathogen isolate. Leaves from $V$. vinifera cv. Muscat Ottonel, V. riparia cv. Gloire de Montpellier, $M$. rotundifolia cv. Carlos, and from the herbaceous species B. vulgaris, L. sativa L. cv. Batavia, and Capsicum sp. were harvested from plants grown in 5 liter pots in the greenhouse. All leaves were rinsed with distilled water and dried with filter paper. Leaf disks of $11 \mathrm{~mm}$ diameter were excised with a cork borer on a PVC pad. They were placed with the abaxial face up in petri dishes on a filter paper wetted with $4 \mathrm{ml}$ of sterile distilled water.

The isolate of $P$. viticola was harvested in the experimental vineyard plot at INRA-Colmar in 2002 on infected $V$. vinifera $\mathrm{cv}$. Chasselas. The inoculum was monosporangial and was replicated weekly on 2-month-old $V$. vinifera cv. Muscat Ottonel shoots grown in a climate chamber. Seven days after inoculation, infected leaves were soaked in sterile distilled water to prepare the sporangia suspension $(50,000$ sporangia/ml $)$ for inoculation on the leaf disks. Infection was initiated by deposing three $10-\mu 1$ drops on the abaxial surface of each leaf disk. The disks were maintained in a humid chamber in petri dishes in a culture room at $21^{\circ} \mathrm{C}$ in the dark until the drops were removed with a vacuum pump $24 \mathrm{~h}$ postinoculation (hpi). Then, the plates were placed under light with a photoperiod of 16/8 h (light/dark, respectively). Progression of infection was stopped by freezing the samples at $-20^{\circ} \mathrm{C} 16$ hpi at 1 day, 3 days, and 5 days postinoculation (dpi). For each genotype, inoculations were performed three times on at least three disks per time point.

Microscopy. After reaching room temperature, frozen specimens were stained with the optical brightener Blankophor as described by Díez-Navajas et al. (4). The presence or absence of encysted zoospores was noted using a light microscope at $\times 25$ magnification and an ocular field observed under these conditions will be referred to as the surface unit. Substomatal vesicle, substomatal vesicle with primary hypha, and long primary hypha are $P$. viticola developmental stages described by Unger et al. (34). These intercellular infection structures were observed on previously Blankophor-stained samples, by using the $\mathrm{KOH}$-aniline blue fluorescence method (11) with some modifications (4). Images were taken with a Canon EOS $300 \mathrm{D}$ camera and processed in a personal computer with the ImageJ program package. For the same $(x, y)$-coordinate, several $z$-coordinate images were taken and a stack was composed with all of them to obtain the definitive microphotograph. At least three disks of each specimen were observed to assure reproducibility.

\section{RESULTS}

At $1 \mathrm{dpi}$, after Blankophor staining $P$. viticola encysted zoospores were often observed near or in stomata of both host ( $V$. vinifera, V. riparia, and $M$. rotundifolia [Fig. 1A through C]) and nonhost species (B. vulgaris, C. annuum, and L. sativa [Fig. 1D through F]). Several zoospores were observed on each infected stomata (2 to 4, sometimes more) for all plant species except for $M$. rotundifolia and $V$. riparia. These two species mostly presented only one zoospore per stomata but the number of infected stomata per surface unit was higher than in $V$. vinifera (A. M. Díez-Navajas, unpublished data). Sometimes on $L$. sativa leaf disks, encysted zoospores with a wandering germ tube were also observed near the leaf veins and not within stomata, probably due to weaker stomatal targeting in this plant species.

After $\mathrm{KOH}$ treatment and aniline blue staining of plant tissues, early developing intercellular structures of $P$. viticola could be observed by epifluorescence in all the inoculated plant species. The substomatal vesicles with primary hyphae were produced in every infection site at $1 \mathrm{dpi}$ (Fig. $1 \mathrm{G}$ through L). Primary elongated hyphae were observed in both Vitis species. In V. vinifera (Fig. 1G) and V. riparia (Fig. 1H) they were much longer than those produced in M. rotundifolia (Fig. 1I). At this timepoint and only in grapevine species, small swollen structures were observed along the hyphae or near the hyphal tips, which were identified as haustoria (Fig. 1I).

At 3 dpi, a complex mycelium with many haustoria was already present in susceptible $V$. vinifera (Fig. $1 \mathrm{M})$. In $V$. riparia, branched hyphae with some haustoria were appearing (Fig. 1N), whereas in $M$. rotundifolia the hyphae were still nonbranched and were a little longer than at $1 \mathrm{dpi}$, although they had several haustoria (Fig. 1O). The frequencies of $P$. viticola infection structures per surface unit were 10 - to 20 -fold lower in the resistant grapevine species ( $V$. riparia and $M$. rotundifolia) than in susceptible $V$. vinifera (A. M. Díez-Navajas, unpublished data). No obvious signs of a defense response induced in the infected host tissues were noted. No haustoria were observed in the nonhost genotypes, and clear signs of defence reaction were visible near the hyphal tips, as highly fluorescent spots corresponding to callose depositions, most likely resulting from the expression of nonhost resistance factors triggered by the incipient haustoria entering mesophyll cells (Fig. 1P through R).

The main change observed between 3 and 5 dpi concerned $V$. vinifera leaf disks, where sporangia appeared, signifying the end of the successful infection cycle (Fig. 1S). In V. riparia leaf disks, a few bundles of branched hyphae able to produce sporangiophores were observed (Fig. 1T). No further progression of infection structures in $M$. rotundifolia were noted, indicating that the life cycle did not progress beyond the elongated hypha stage (Fig. 1U). In the three nonhost species, pathogen growth was contained by the plant defense system before the primary haustorium was established (Fig. 1V through Y). Haustoria were observed in $M$. rotundifolia (Fig. 1U) whereas such structures were never observed in the nonhost species (Fig. 1V through Y).

\section{DISCUSSION}

During the $P$. viticola life cycle, stomata play an important role for the infection and sporulation stages $(1,6)$. Concerning the infection step, stomata represent natural openings providing direct access to the mesophyll for encysted $P$. viticola zoospores. The resistance level could be related to the number of stomata present on leaf surface. The stomatal density of Vitis species ranges between 140 and $300 / \mathrm{mm}^{2}$, whereas the density of $M$. rotundifolia reaches $400 / \mathrm{mm}^{2}$ (27). Thus, it seems that the resistance level of grapevine species is not dependant on the stomatal number. Blankophor-stained samples showed important differences in $P$. viticola zoospore encystment on stomata between host grapevine species and between grapevine and herbaceous nonhost species. The numbers of encysted zoospores per leaf area unit were on average equivalent in all six species but their distribution on the leaf epidermis was different. Clusters of encysted zoospores on stomata were observed in nonhost species as well as in 
the susceptible host $V$. vinifera, but rarely on the two resistant grapevine species. If $P$. viticola zoospores are targeted by host factors released by open stomata (17), it can be suggested that the lesser attractiveness of individual $M$. rotundifolia and $V$. riparia stomata could be balanced by a higher stomatal aperture. All these observations indicate that the signals or factors expected to drive P. viticola zoospores to stomata are not specific to Vitis, Muscadinia species, or nonhost species. Such nonspecific tactic responses have already been observed for other phytopathogenic oomycetes $(33,36)$. Chemo-attractive plant factors such as amino

\section{Encysted zoospores}
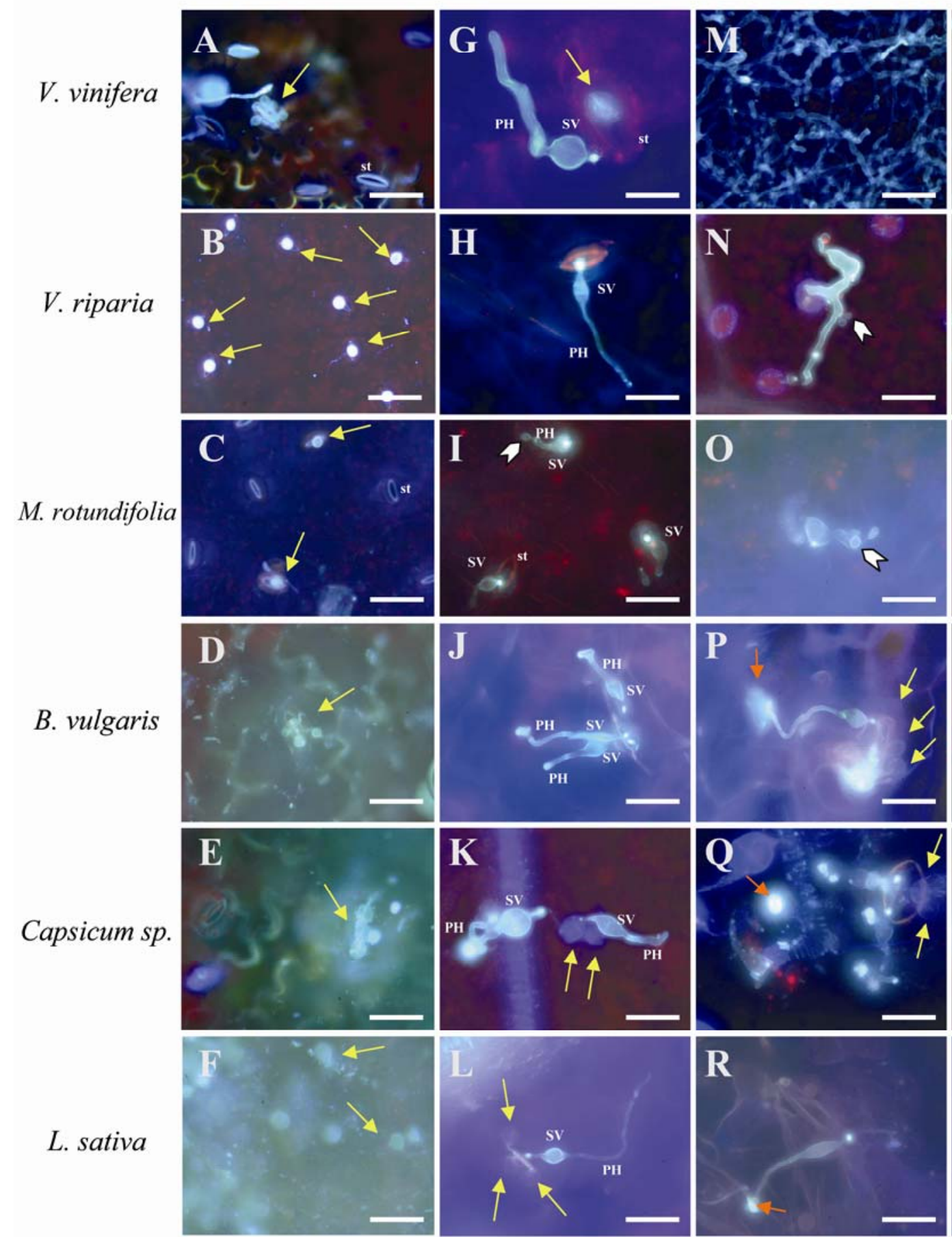

\section{Capsicum sp}

\section{L. sativa}

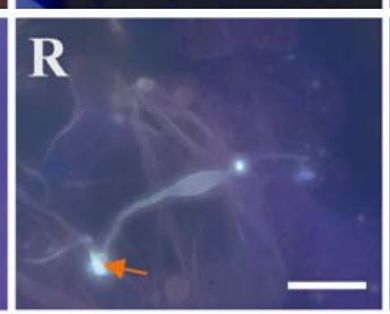

3 dpi

5 dpi
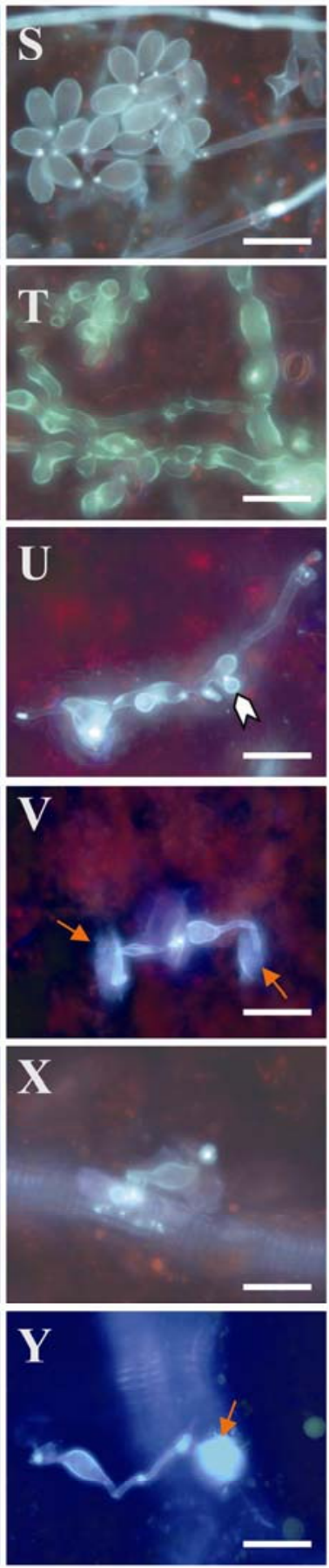

Fig. 1. Plasmopara viticola encysted zoospores on stomata (st) were observed in Vitis vinifera (A), V. riparia (B), Muscadinia rotundifolia (C), Beta vulgaris (D), Capsicum sp. (E), and Lactuca sativa (F) after Blankophor staining. After penetration of the germ tube in the stomata, all further intercellular infection structures were observed using aniline blue staining. At 1 day postinoculation (dpi), the substomatal vesicle (SV) and primary hypha (PH) were visible in all the specimens (G to L). At 3 dpi, a mycelium was already visible in V. vinifera (M), elongated and branched hyphae with haustoria were observed in V. riparia (N), primary haustoria were seen in $M$. rotundifolia $(\mathbf{O})$ and only primary hyphae were observed in B. vulgaris $(\mathbf{P})$, Capsicum $\mathrm{sp}$. (Q), and $L$. sativa $(\mathbf{R})$. At 5 dpi, in $V$. vinifera (S) aerial sporangiophores were produced from invaded stomata, while a small mycelium could occasionally be observed in $V$. riparia (T), an elongated hypha with haustoria was present in M. rotundifolia (U) and a blocked hypha bordered with accumulating fluorescent compounds was seen in B. vulgaris (V), Capsicum sp. $(\mathbf{X})$, and $L$. sativa $(\mathbf{Y})$. Yellow arrows: encysted zoospores; thick arrowheads: haustoria; orange arrows: possible glucan accumulations (callose). Bars: $10 \mu \mathrm{m}$ (R), $20 \mu \mathrm{m}(\mathbf{G}$ to $\mathbf{K}, \mathbf{O}$ to $\mathbf{Q}, \mathbf{U}$ to $\mathbf{Y}), 40 \mu \mathrm{m}(\mathbf{A}$ to $\mathbf{F}, \mathbf{N}, \mathbf{T})$, and $100 \mu \mathrm{m}(\mathbf{M}, \mathbf{S})$. 
acids, pectins (20), and isoflavones (23) have been identified. These substances are not considered specific to the pathogen host range but are able to direct zoospores to defined sites on the plant that favor the entry of the parasites according to their penetration mode (8). This general effect of plant factors on $P$. viticola zoospores implies that the obligate biotrophic interactions with grapevine cells take place later in the infection cycle, when the pathogen actively penetrates the plant cell wall for access to intracellular nutrients.

For some oomycetes like Phytophthora and Pythium species as well as for rust and powdery fungi, penetration of the plant surface corresponds to the penetration of the epidermic cell wall. These pathogens produce an appressorium from which a penetration peg develops to penetrate the epidermal cell wall and to elongate into an intracellular infection hypha. For $P$. viticola and other downy mildews, zoospores attach to the stomata where they encyst and develop a germ tube that grows into the substomatal cavity. After formation of a septum at the apex of the germ tube, a substomatal vesicle develops followed by the growth of the primary hypha (17). The first real plant cell-pathogen interaction is delayed until the primary hypha reaches a mesophyll cell.

In incompatible and nonhost interactions, cell wall-associated defense responses are triggered when penetration starts $(9,12)$. Cytoplasmic aggregation and cell wall appositions successfully inhibit pathogen growth in nonhost interactions (8). In the case of $P$. viticola, the first signs of response from attacked nonhost tissue appear at the formation of the primary hypha, probably when haustoria started to enter mesophyll cells. Mature haustoria were never observed in the nonhost species. In nonhost species, the attempted penetration is contained by the immediate triggering of cell wall-associated defense responses leading to callose depositions at the sites of haustorium formation. Although callose deposition is generally revealed by aniline blue staining, it would nevertheless be of interest to carry out a more detailed study of sites of deposition by immunocytochemistry. It is thought that callose deposition functions as a mechanical barrier to limit pathogen growth by conferring greater strength to the host cell wall (28). Such resistance is described as type I nonhost resistance and is probably the most common type of nonhost resistance (22). In type I nonhost resistance, the nonhost pathogen is not able to overcome the constitutive and general elicitor-induced plant defense responses such as cell wall thickening and secondary metabolites accumulation (22).

Concerning nonhost resistance of grapevine, the only example described concerns interaction with a nonhost downy mildew oomycete (Pseudoperonospora cubensis). When grapevine leaves were inoculated with the nonhost oomycete, they exhibited faster defense responses than against $P$. viticola, independent of the susceptibility or the resistance to downy mildew of the grape cultivar (18). It is reasonable to hypothesize that $P$. cubensis is able to initiate an infection of grape tissue up to the stage of first haustorium formation, which then induces the rapid activation of defense genes in infected cells in a more efficient way than during $P$. viticola haustoria production.

During host resistance, the pathogen is able to overcome the first line of constitutive and general elicitor-induced plant defense responses. Cell wall disruption due to the action of cell walldegrading secretome then leads to haustoria formation. The interesting feature at the cellular level is that in both resistant and susceptible grapevine tissues, fully developed haustoria are visible, which indicates that activation of the defense responses depends on cytoplasmic effectors produced by operational $P$. viticola haustoria. Necrosis is a typical response to $P$. viticola infection in resistant grapevines. In totally resistant species like $M$. rotundifolia, a hypersensitive response (HR) is observed around infected stomata several hours after inoculation (S. Wiedemann-Merdinoglu, personal communication) which sometimes progresses to necrotic points (3). In partially resistant species like V. rupestris $(3,34)$ as well as in the partially resistant cultivar Solaris (6), necrosis appears several days after infection as necrotic spots. In spite of the necessary delay for haustorium recognition, a classical incompatible interaction would happen in both resistant species, leading to the death of the first cells in contact with haustoria and the end of the hyphal growth. Concerning different resistant host species, the differences of resistance level could be explained by a variation in efficiency of the HR. The time course of HR onset as measured using the diaminobenzidine (DAB) method (32) would give a better view of the importance of HR in containing $P$. viticola development among different resistant host species.

In conclusion, $P$. viticola seems to modulate host cell defenses through apoplastic and cytoplasmic effectors secreted during the first stages of infection on nonhost and host plants. Apoplastic effectors are known to be secreted into the plant extracellular space and interact with plant cell surface receptors. This interaction triggers rapid defense responses. When $P$. viticola interacts with the cell wall of a nonhost plant, the plant probably responds to these general effectors so as to avoid further haustorium development. Cytoplasmic effectors are translocated inside the plant cell through haustoria and interact with cytoplasmic targets leading to susceptibility or resistance according to the nature of the host $(2,16)$. Our findings emphasize thus the central role of haustoria both in the establishment of biotrophic parasitism and in the induction of the hypersensitive response in resistant grapevine genotypes.

\section{ACKNOWLEDGMENTS}

We thank P. Mestre and L. Schmidlin for critical reading of the manuscript and P. Coste for technical assistance in plant and inoculum maintenance. We also thank K. E. Richards for improving the English of the manuscript. A. M. Díez thanks the Departamento de Educación, Universidades e Investigación del Gobierno Vasco-Eusko Jaurlaritza for financial support by a postdoctoral fellowship.

\section{LITERATURE CITED}

1. Allègre, M., Daire, X., Héloir, M.-C., Trouvelot, S., Mercier, L., Adrian, M., and Pugin, A. 2007. Stomatal deregulation in Plasmopara viticolainfected grapevines leaves. New Phytol. 173:832-840.

2. Birch, P. R. J., Rehmany, A. P., Pritchard, L., Kamoun, S., and Beynon, J. L. 2006. Trafficking arms: Oomycete effectors enter host plant cells. Trends Microbiol. 14:8-11.

3. Dai, G. H., Andary, C., Mondolot-Cosson, L., and Boubals, D. 1995. Histochemical studies on the interaction between three species of grapevine, Vitis vinifera, $V$. rupestris and $V$. rotundifolia and the downy mildew fungus, Plasmopara viticola. Physiol. Mol. Plant Pathol. 46:177188.

4. Díez-Navajas, A. M., Greif, C., and Merdinoglu, D. 2007. Two simplified fluorescent staining techniques to observe infection structures of the oomycete Plasmopara viticola in grapevine leaf tissues. Micron 38:680683.

5. Eichmann, R., and Hückelhoven, R. 2008. Accommodation of powdery mildew fungi in intact plant cells. J. Plant Physiol. 165:5-18.

6. Gindro, K., Pezet, R., and Viret, O. 2003. Histological study of the responses of two Vitis vinifera cultivars (resistant and susceptible) to Plasmopara viticola infections. Plant Physiol. Biochem. 41:846-853.

7. Goldy, R. G., and Onokpise, O. U. 2001. Genetics and breeding. Pages 51-90 in: Muscadine Grapes. F. M. Basiouny and D. G. Himelrick, eds. ASHS Press, Alexandria, VA.

8. Hardham, A. R. 2007. Cell biology of plant-oomycete interactions. Cell. Microbiol. 9:31-39.

9. Heath, M. C. 2002. Cellular interactions between biotrophic fungal pathogens and host or nonhost plants. Can. J. Plant Pathol. 24:259-264.

10. Heath, M. C., and Skalamera, D. 1997. Cellular interactions between plants and biotrophic fungal parasites. Adv. Bot. Res. 24:195-225.

11. Hood, M. E., and Shew, H. D. 1996. Applications of KOH-aniline blue fluorescence in the study of plant-fungal interactions. Phytopathology 86:704-708.

12. Hückelhoven, R. 2007. Cell wall-associated mechanisms of disease resistance and susceptibility. Annu. Rev. Phytopathol. 45:101-127.

13. Humphry, M., Consonni, C., and Panstruga, R. 2006. Mlo-based powdery mildew immunity: Silver bullet or simply non-host resistance? Mol. Plant 
Pathol. 7:605-610

14. Jones, J. D. G., and Dangl, J. L. 2006. The plant immune system. Nature 444:323-329.

15. Kamoun, S. 2001. Nonhost resistance to Phytophthora: Novel prospects for a classical problem. Curr. Opin. Plant Biol. 4:295-300.

16. Kamoun, S. 2006. A catalogue of the effector secretome of plant pathogenic oomycetes. Annu. Rev. Phytopathol. 44:41-60.

17. Kiefer, B., Riemann, M., Büche, C., Kassemeyer, H. H., and Nick, P. 2002. The host guides morphogenesis and stomatal targeting in the grapevine pathogen Plasmopara viticola. Planta 215:387-393.

18. Kortekamp, A. 2006. Expression analysis of defence-related genes in grapevine leaves after inoculation with a host and a non-host pathogen. Plant Phys. Biochem. 44:58-67.

19. Latijnhouwers, M., De Wit, P. J. G. M., and Govers, F. 2003. Oomycetes and fungi: similar weaponry to attack plants. Trends Microbiol. 11:462469.

20. Leano, E. M., Vrijmoed, L. L. P., and Jones, E. B. G. 1998. Zoospore chemotaxis of two mangrove strains of Halophytophthora vesicula from Mai Po Hong Kong. Mycologia 90:1001-1008.

21. Mendgen, K., and Hahn, M. 2002. Plant infection and the establishment of fungal biotrophy. Trends Plant Sci. 7:332-356.

22. Mysore, K. S., and Ryu, C. M. 2004. Nonhost resistance: How much do we know? Trends Plant Sci. 9:97-104.

23. Morris, P. F., and Ward, E. W. B. 1992. Specificity in the chemoattraction of zoospores of Phytophthora sojae by soybean isoflavones. Can. J. Plant Pathol. 14:246.

24. Panstruga, R. 2003. Establishing compatibility between plants and obligate biotrophic pathogens. Curr. Opin. Microbiol. 6:320-326.

25. Schulze-Lefert, P. 2004. Knocking on the heaven's wall: Pathogenesis of and resistance to biotrophic fungi at the cell wall. Curr. Opin. Plant Biol. $7: 377-383$.
26. Schweizer, P. 2007. Nonhost resistance of plants to powdery mildewNew opportunities to unravel the mystery. Phys. Mol. Plant Pathol. 70:3-7.

27. Shiraishi, S., Hsiung, T., and Shiraishi, M. 1996. Preliminary survey on stomatal density and length of grapevine. J. Fac. Agric., Kyushu Univ., 41:11-15.

28. Soylu, E. M., Soylu, S., and Mansfield, J. W. 2004. Ultrastructural characterization of pathogen development and host responses during compatible and incompatible interactions between Arabidopsis thaliana and Peronospora parasitica. Phys. Mol. Plant Pathol. 65:67-78.

29. Spencer, D. M. 1981. Pages 636 in: The Downy Mildews. Academic Press, London, UK.

30. Staudt, G., and Kassemeyer, H. H. 1995. Evaluation of downy mildew resistance in various accessions of wild Vitis species. Vitis 34:225-228.

31. Thordal-Christensen, H. 2003. Fresh insights into processes of nonhost resistance. Curr. Opin. Plant Biol. 6:351-357.

32. Thordal-Christensen, H., Zhang, Z., Wei, Y., and Collinge, D. B. 1997. Subcellular localization of $\mathrm{H}_{2} \mathrm{O}_{2}$ in plants. $\mathrm{H}_{2} \mathrm{O}_{2}$ accumulation in papillae and hypersensitive response during the barley-powdery mildew interaction. Plant J. 11:1187-1194.

33. Tyler, B. M. 2002. Molecular basis of recognition between Phytophthora pathogens and their hosts. Annu. Rev. Phytopathol. 40:137-167.

34. Unger, S., Büche, C., Boso, S., and Kassemeyer, H. H. 2007. The course of two different Vitis genotypes by Plasmopara viticola indicates compatible and incompatible host-pathogen interactions. Phytopathology 97:780-786.

35. Vleeshouwers, V. G. A. A., Van Dooijeweert, W., Govers, F., Kamoun, S., and Colon, L. T. 2000. The hypersensitive response is associated with host and nonhost resistance to Phytophthora infestans. Planta 210:853864.

36. Walker, C. A., and Van West, P. 2007. Zoospore development in the oomycetes. Fungal Biol. Rev. 21:10-18. 